\title{
Papiloma invertido de tracto urinario superior
}

\author{
R. Ferrero Doria, E. Huertas Valero*, B. Coronel Sánchez, F. Moreno Pérez, F. García Víctor, \\ E. Díaz Calleja
}

Servicio de Urología. *Servicio de Anatomía Patológica. Hospital Francesc Borja. Gandía (Valencia).

Actas Urol Esp 2005; 29 (4): 423-426

\section{RESUMEN}

PAPILOMA INVERTIDO DE TRACTO URINARIO SUPERIOR

El papiloma invertido del tracto urinario superior es una lesión rara y considerada generalmente de comportamiento benigno. Presentamos dos casos de papiloma invertido de uréter, descubiertos tras estudio por infección urinaria alta y hematuria macroscópica respectivamente. Los dos asociaron carcinomas vesicales asincrónicos y sincrónicos durante su seguimiento, lo que nos obliga a realizar un seguimiento estricto.

Palabras clave: Uréter. Tracto urinario alto. Papiloma invertido. Tumores del TUS.

\section{ABSTRACT \\ INVERTED PAPILLOMA OF THE UPPER URINARY TRACT}

Inverted papilloma of the upper urinary tract is a rare tumor and generally considered to be a benign. We present two case of benign ureteral inverted papiloma. They were discovered after to study a upper urinary infection and a gross hematuria respectively. Both cases appear to be associated with asynchronous and synchronous bladder carcinoma, so it is very important a strict follow-up.

Keywords: Ureter. Upper tract urinary. Inverted papiloma. Upper GU tract tumors.

$\mathrm{M}$ uchos han sido los papilomas invertidos (PI) uroteliales descritos desde que en 1963 Potts y Hirst ${ }^{1}$ acuñaran dicho término. La gran mayoría de ellos se ubican en la vejiga y tienen un comportamiento benigno. Muchos menos casos han sido descritos en la literatura que asiente en el tracto urinario superior y menos aún que este PI tenga en su interior una transformación maligna carcinomatosa que ya no lo hace considerar como de comportamiento benigno. Presentamos dos casos de PI diagnosticados y tratados en nuestro centro, detallando cual ha sido nuestra actitud y seguimiento así como el comportamiento y la evolución de los mismos.

\section{CASOS CLÍNICOS}

Caso Clínico 1 (S.C.B.)

Varón de 63 años que ingresa por urgencias por clínica compatible con Pielonefrítis aguda izquierda. Antecedentes de cólicos nefríticos izquierdos no estudiados y hepatitis en la juventud. En la exploración destaca una puñopercusión renal izquierda positiva y un tacto rectal con hipertrofia prostática volumen III/IV, fibroadenomatosa y sin nódulos. Analítica sanguínea con bioquímica dentro de la normalidad, PSA de $3,4 \mathrm{ng} / \mathrm{ml}$ y formula sanguínea con leucocitosis y desviación izquierda. Analítica de orina con nitritos positivos y leucocituria. Urocultivos y Hemocultivos positivos a E. Coli. 
Rx simple de aparato urinario sin hallazgos patológicos significativos. Ecografía con ectasia renal izquierda grado II-III. La UIV realizada muestra vía urinaria alta derecha dentro de la normalidad y retraso en la captación y eliminación de contraste del riñón izquierdo que persiste a las dos horas. Se procede a la realización de nefrostomía derivativa izquierda y posterior pielografía anterógrada (Fig. 1), la cual demuestra defecto de repleción en uréter iliaco compatible con tumoración ureteral de 3-4 cm y uréter en anzuelo. Citología a través de la nefrostomía negativa y el estudio de extensión con TAC objetivaba dicha lesión como confinada al órgano origen. Se realizó Nefroureterectomía izquierda previa desinserción endoscópica, siendo el resultado anatomopatológico de Papiloma invertido de uréter (Fig. 2) y riñón izquierdo con pielonefritis crónica. A los dos años el paciente sufre una RAO que aconseja la realización de Adenomectomía Retropúbica y posteriormente a los 4 y 5 años presenta sendas
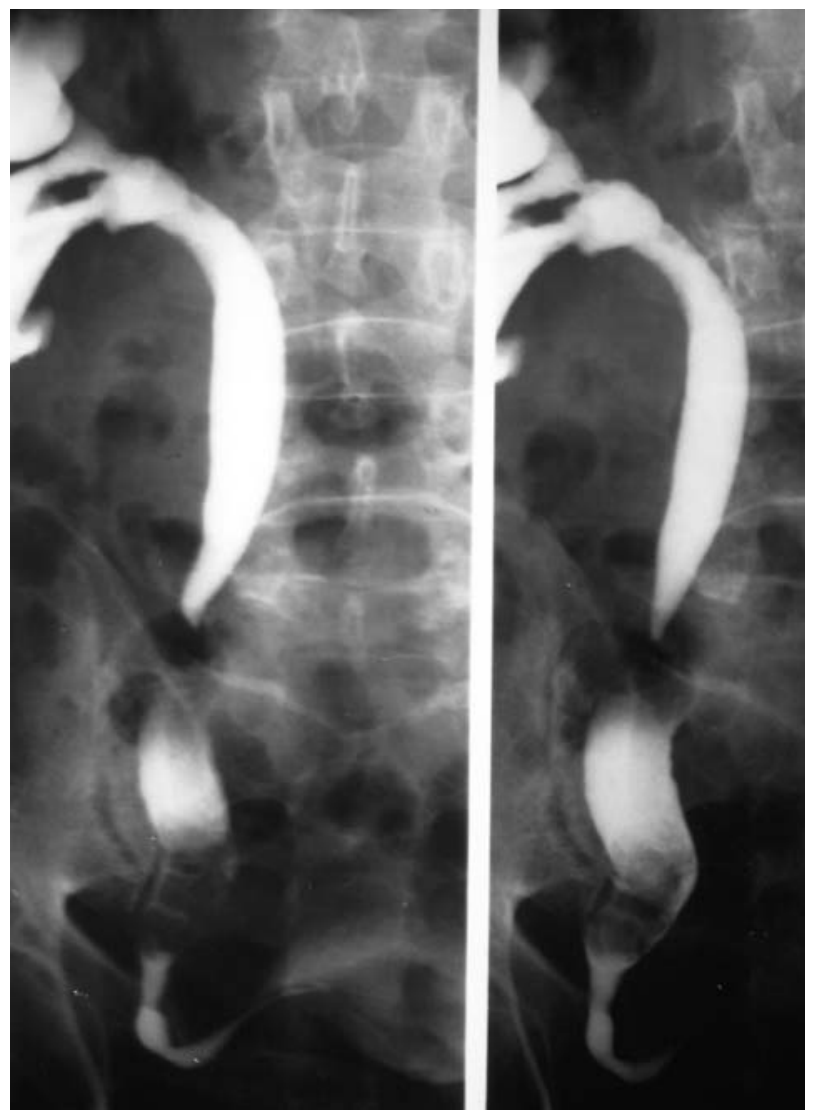

FIGURA 1. Pielografía anterógrada izquierda: defecto repleción en uréter iliaco izquierdo.

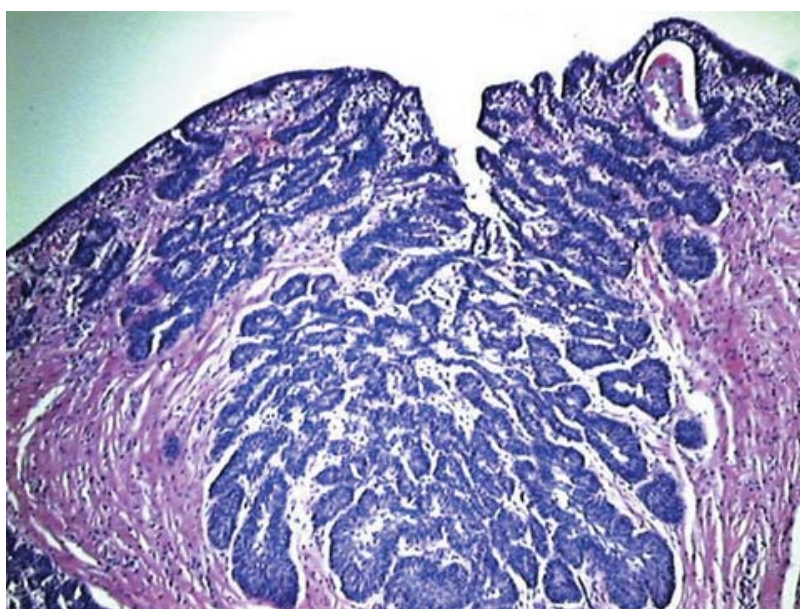

FIGURA 2. Preparación patológica: papiloma invertido.

recidivas vesicales de Carcinoma transicional de vejiga T1GII. El paciente sigue vivo libre de enfermedad urotelial a los 6 años del diagnóstico de PI.

\section{Caso Clínico 2 (A.C.R.)}

Varón de 65 años que consulta por hematuria total macroscópica sin coágulos. Antecedentes de hiperlipemia, ulcus peptico y cardiopatía isquémica. Exploración: hernia inguinal bilateral, genitales externos dentro de la normalidad, tacto

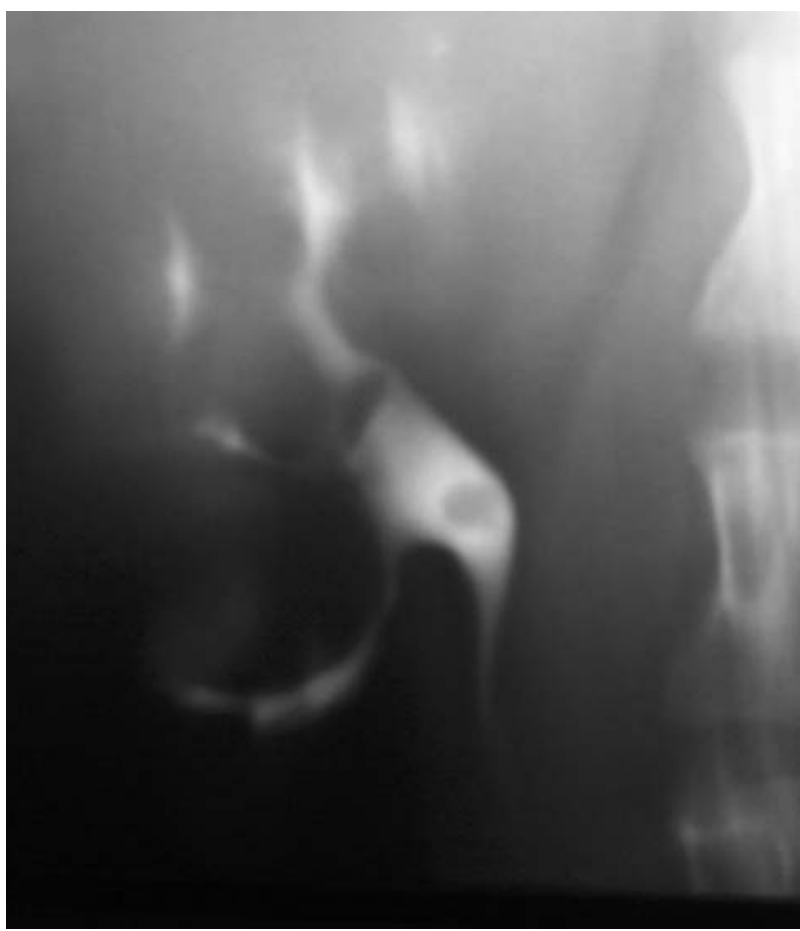

FIGURA 3. Urografia intravenosa: defecto repleción central en pelvis renal derecha. 
rectal con hipertrofia prostática volumen II/IV fibroblástica y sin nódulos. Analítica sanguínea normal. UIV: defecto de repleción en pared lateral derecha de la vejiga y otro en pelvis renal derecha (Fig. 3). Citología urinaria selectiva de vía urinaria alta derecha negativa para células tumorales. Se realiza RTU de neoformación vesical y Pielectomía parcial derecha con resultado anatomopatológico de Carcinoma transicional de vejiga T1 GII y papiloma invertido de pelvis renal (Fig. 4) respectivamente. En el seguimiento, con cistoscopias, citologías y UIV, el paciente presenta al año y a los tres años recidiva vesical de carcinoma transicional (T1GII). A los 6 años del diagnóstico inicial el paciente presenta metástasis en cavidad craneal y pared abdominal de carcinoma de células pequeñas cuyo órgano de origen es desconocido, falleciendo a los 3 meses de éste último diagnóstico.

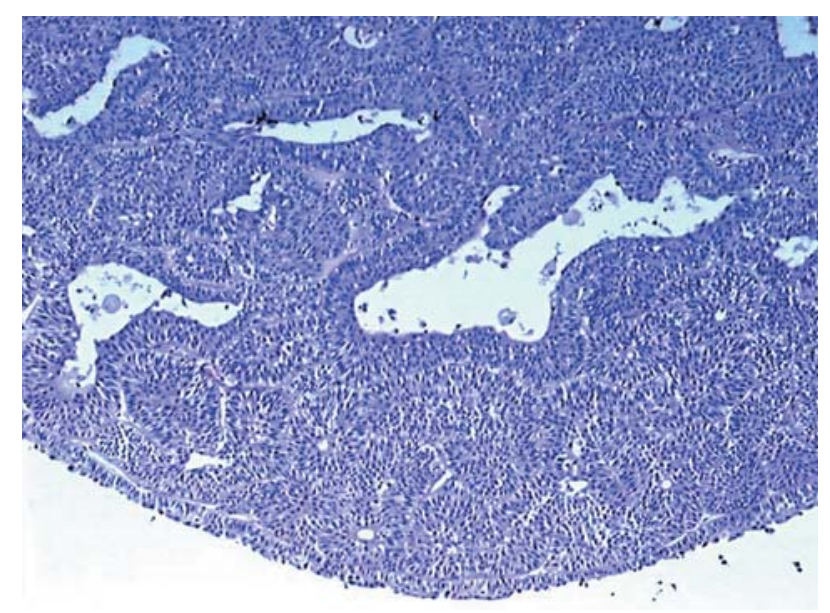

FIGURA 4. Preparación patológica: papiloma invertido.

\section{DISCUSIŌN}

Fueron Potts y Hirst ${ }^{1}$ quienes en 1963 describieron por primera vez el término de papiloma invertido que histológicamente presenta un crecimiento papilar en dirección al intersticio de la submucosa. Mayoritariamente estos tumores han sido descritos en la vejiga, aunque también se describen en el tracto urinario superior ${ }^{2}$ y en la uretra $^{3}$. La incidencia de estos PI varian según las series desde un $2,2^{2}$ a un $1.6 \% \%^{4}$ de los tumores del tracto urinario. Es posible que algunos tumores uroteliales grado I hallan sido diagnosticados de forma errónea y sean realmente papilomas invertidos, y esto se debe por la dificultad de encontrar diferencias entre ambos ${ }^{4}$. El 90\% de los pacientes son varones entre la sexta y séptima década de la vida ${ }^{5}$. La hematuria macroscópica es el síntoma más común ${ }^{5}$, manifestándose también con dolor lumbar y síntomas obstructivos ${ }^{6}$.

El estudio patológico ${ }^{5}$ revela lesión papilar, pediculada, de superficie lisa y generalmente única. El estudio microscópico demuestra un urotelio típico que recubre la superficie externa de la lesión, de la cual surgen cordones invertidos en profundidad, asentando sobre ejes de estroma fibrovascular. Los PI benignos presentan una membrana basal intacta sin figuras mitóticas. También han sido descritos en el estroma focos de células inflamatorias de cistitis quística o glandular, así como nidos de Von Brunn.

Etiológicamente el origen de estas lesiones es desconocido. Se ha sugerido que el PI puede desarrollarse desde una proliferación de cistitis quística o glandular por metaplasia de nidos de Von Brunn ${ }^{7}$, hasta incluso ser consideradas como verdaderas neoplasias ${ }^{4}$.

$\mathrm{El}$ pronóstico de estos tumores en general es benigno. Han sido documentados casos con invasión superficial del estroma ${ }^{7}$ pero no con metástasis. Aún así si han sido descritos, en la literatura, PI con componente maligno y por lo tanto con potencial invasión y metástasis ${ }^{4,5,7,8}$ y cuyo reconocimiento ha de ser preciso, sobre todo para correcto y estricto seguimiento del mismo.

El diagnóstico de estas lesiones resulta del estudio de la hematuria o síntomas obstructivo, forma en la que se manifiesta. Por ello la UIV, con o sin pielografias, objetiva el defecto de repleción que nos pone en alerta. Habitualmente nos solemos apoyar con la citología urinaria, de micción espontánea o selectiva de la vía urinaria afecta, así con estudios de extensión con el TAC. La cistoscopia es útil para descartar lesiones de localización vesical con las que se pueda acompañar estos tumores del tracto urinario superior ${ }^{9}$ (como uno de nuestros casos). La utilización de ureterorrenoscopia diagnóstica puede permitir un mejor diagnóstico ${ }^{6,10}$ y ayudarnos a planificar una cirugía conservadora.

El tratamiento más empleado en este tipo de lesiones de la vía urinaria alta ha sido la Nefroureterectomía dado que, inicialmente y por 
frecuencia, la lesión maligna es la primera consideración que se tiene en mente ante el diagnóstico de una lesión tumoral de estas características $^{4}$. También han sido tratados con técnicas quirúrgicas abiertas conservadoras como la ureterorrafia término-terminal, pielectomía (como uno de nuestros casos). Si bien, con el empleo de las últimas tecnologías como es la ureterorrenoscopia, es también posible el tratamiento endoscópico $^{6}$.

Desde 1980 han sido recogidos casos de PI con recurrencias y complicados con carcinoma de células transicionales, lo cual encuadra a esta entidad en un contexto algo sombrío. Algunos trabajos constatan las recurrencias, malignizaciones parciales y complicaciones con cáncer ${ }^{11}$. Nuestros dos casos de PI de la vía urinaria alta se han asociado a carcinomas transicionales superficiales de forma sincrónica o asincrónica. Todo ello obliga a un seguimiento lo suficientemente estricto ante la posibilidad de recurrencia de este mismo tumor o de una forma maligna en cualquier parte del urotelio.

\section{REFERENCIAS}

1. Potts IF, Hirst E. Inverted papiloma of the bladder. J Urol 1963;90:175.

2. Kunze E, Schauer A, Schmitt M. Histology and histogenesis of two different types of inverted urothelial papillomas. Cancer 1983;51(2):348-358.
3. Lausten GS, Anagnostaki L, Thomsen OF. Inverted papiloma of the upper urinary tract. Eur Urol 1984; 10:67.

4. Asano K, Miki J, Maeda S, Naruaka T, Takahashi H, Oishi Y. Clinical studies on inverted papilloma of urinary tract: report of 48 cases and review of the literature. J Urol 2003 Oct;170(4 Pt 1):1209-1212.

5. Kyriakos M, Royce RK. Multiple simultaneous inverted papillomas of the upper urinary tract. Cancer 1989 Jan 15; 63(2):368-380.

6. Cabezas Zamora M, Iglesias JL, Pérez-Castro E, Mancebo JM, Massarra J. Papiloma invertido de uréter. Presentación excepcional y revisión de la literatura. Arch Esp Urol 1989 Jul-Aug;42(6) 564-567.

7. Grainger R, Gikas PW, Grossman HB. Urothelial carcinoma occurring within an inverted papiloma of the ureter. $\mathrm{J}$ Urol 1990 Apr; 143(4):802-804.

8. Stower MJ, M Maclver AG, Gingell JC, Clarke E. Inverted papiloma of the ureter with malignant change. $\mathrm{Br} \mathrm{J}$ Urol 1990 Jan;65(1):13-16.

9. Martín-Laborda y Bergasa F, Virseda Chamorro M, Bueno Sánchez JL, Vallejo Herrador J, Coca Mechero S. Papiloma invertido de uréter. Revisión y criterio de tratamiento. Arch Esp Urol 1990 Sep;43(7):749-754.

10. Giménez Blasco N, Sanz Vélez JI, Esclarin Duny MA, Marigil Gómez M. Papiloma invertido de uréter. A propósito de un caso. Actas Urol Esp 1998 apr;22(4):370-373.

11. Mattelaer J, Leonard A, Goddeeris P, D`Hoedt M, Van Kerrebroeck P. Inverted papiloma of bladder: clinical significance. Urology 1988,32:192.

Dr. R. Ferrero Doria

Avda. Albaida, 2, escalera 1, pta. 18.

46870 Ontinyent (Valencia)

(Trabajo recibido el 1 julio de 2004) 